Ann. Funct. Anal. 7 (2016), no. 2, 314-325

http://dx.doi.org/10.1215/20088752-3506016

ISSN: 2008-8752 (electronic)

ANNALS of

EUNCTIONAL

http://projecteuclid.org/afa

ANALYSIS

\title{
A CHARACTERIZATION OF THE RIESZ FAMILY OF SHIFTS OF FUNCTIONS ON LOCALLY COMPACT ABELIAN GROUPS
}

\author{
N. MOHAMMADIAN,${ }^{1 *}$ R. A. KAMYABI GOL,${ }^{2}$ and R. RAISI TOUSI ${ }^{2}$ \\ Communicated by V. Runde
}

\begin{abstract}
For a locally compact Abelian group $G$, we give a necessary and sufficient condition for shifts of a function $\phi \in L_{2}(G)$ to be a Riesz family. Also, for a finite family $\Phi$ of compactly supported functions in $L_{2}(G)$, we show that the shifts of $\Phi$ constitute a Riesz family if and only if the nets $(\phi(\xi \eta))_{\eta \in L^{\perp}}$, $\phi \in \Phi$, are linearly independent for all $\xi \in \hat{G}$.
\end{abstract}

\section{INTRODUCTION AND PRELIMINARIES}

Shift-invariant spaces and Riesz families play an increasingly important role in various areas of mathematical analysis and their applications. They appear in the study of spline wavelets, approximation, regular sampling, Gabor systems, and several others (see [1], [4], [8], [14]). A sequence $\left(x_{n}\right)_{n \in \mathbb{N}}$ for a Hilbert space $H$ is called a Riesz basis if it is a frame and is also a basis for $H$. Frames provide a useful tool to obtain signal decomposition in cases where redundancy, oversampling, and irregular sampling play a role (see [2], [6]). A frame for a vector space equipped with an inner product also allows each vector in the space to be written as a linear combination of the elements in the frame, but linear independence between the frame elements is not required. Intuitively, one can think about a frame as a basis to which one has added more elements (see [5]). One of the main purposes of this paper is to give a necessary and sufficient condition for a finite family of functions to constitute a Riesz family via linear independence of the

Copyright 2016 by the Tusi Mathematical Research Group.

Received Apr. 16, 2015; Accepted Aug. 15, 2015.

${ }^{*}$ Corresponding author.

2010 Mathematics Subject Classification. Primary 43A15; Secondary 43A25.

Keywords. Riesz family, locally compact Abelian group, uniform lattice, shifts of functions. 
Ann. Funct. Anal. 7 (2016), no. 2, 314-325

http://dx.doi.org/10.1215/20088752-3506016

ISSN: 2008-8752 (electronic)

ANNALS of

EUNCTIONAL

http://projecteuclid.org/afa

ANALYSIS

\title{
A CHARACTERIZATION OF THE RIESZ FAMILY OF SHIFTS OF FUNCTIONS ON LOCALLY COMPACT ABELIAN GROUPS
}

\author{
N. MOHAMMADIAN,${ }^{1 *}$ R. A. KAMYABI GOL,${ }^{2}$ and R. RAISI TOUSI ${ }^{2}$ \\ Communicated by V. Runde
}

\begin{abstract}
For a locally compact Abelian group $G$, we give a necessary and sufficient condition for shifts of a function $\phi \in L_{2}(G)$ to be a Riesz family. Also, for a finite family $\Phi$ of compactly supported functions in $L_{2}(G)$, we show that the shifts of $\Phi$ constitute a Riesz family if and only if the nets $(\phi(\xi \eta))_{\eta \in L^{\perp}}$, $\phi \in \Phi$, are linearly independent for all $\xi \in \hat{G}$.
\end{abstract}

\section{INTRODUCTION AND PRELIMINARIES}

Shift-invariant spaces and Riesz families play an increasingly important role in various areas of mathematical analysis and their applications. They appear in the study of spline wavelets, approximation, regular sampling, Gabor systems, and several others (see [1], [4], [8], [14]). A sequence $\left(x_{n}\right)_{n \in \mathbb{N}}$ for a Hilbert space $H$ is called a Riesz basis if it is a frame and is also a basis for $H$. Frames provide a useful tool to obtain signal decomposition in cases where redundancy, oversampling, and irregular sampling play a role (see [2], [6]). A frame for a vector space equipped with an inner product also allows each vector in the space to be written as a linear combination of the elements in the frame, but linear independence between the frame elements is not required. Intuitively, one can think about a frame as a basis to which one has added more elements (see [5]). One of the main purposes of this paper is to give a necessary and sufficient condition for a finite family of functions to constitute a Riesz family via linear independence of the

Copyright 2016 by the Tusi Mathematical Research Group.

Received Apr. 16, 2015; Accepted Aug. 15, 2015.

${ }^{*}$ Corresponding author.

2010 Mathematics Subject Classification. Primary 43A15; Secondary 43A25.

Keywords. Riesz family, locally compact Abelian group, uniform lattice, shifts of functions. 
Ann. Funct. Anal. 7 (2016), no. 2, 314-325

http://dx.doi.org/10.1215/20088752-3506016

ISSN: 2008-8752 (electronic)

ANNALS of

EUNCTIONAL

http://projecteuclid.org/afa

ANALYSIS

\title{
A CHARACTERIZATION OF THE RIESZ FAMILY OF SHIFTS OF FUNCTIONS ON LOCALLY COMPACT ABELIAN GROUPS
}

\author{
N. MOHAMMADIAN,${ }^{1 *}$ R. A. KAMYABI GOL,${ }^{2}$ and R. RAISI TOUSI ${ }^{2}$ \\ Communicated by V. Runde
}

\begin{abstract}
For a locally compact Abelian group $G$, we give a necessary and sufficient condition for shifts of a function $\phi \in L_{2}(G)$ to be a Riesz family. Also, for a finite family $\Phi$ of compactly supported functions in $L_{2}(G)$, we show that the shifts of $\Phi$ constitute a Riesz family if and only if the nets $(\phi(\xi \eta))_{\eta \in L^{\perp}}$, $\phi \in \Phi$, are linearly independent for all $\xi \in \hat{G}$.
\end{abstract}

\section{INTRODUCTION AND PRELIMINARIES}

Shift-invariant spaces and Riesz families play an increasingly important role in various areas of mathematical analysis and their applications. They appear in the study of spline wavelets, approximation, regular sampling, Gabor systems, and several others (see [1], [4], [8], [14]). A sequence $\left(x_{n}\right)_{n \in \mathbb{N}}$ for a Hilbert space $H$ is called a Riesz basis if it is a frame and is also a basis for $H$. Frames provide a useful tool to obtain signal decomposition in cases where redundancy, oversampling, and irregular sampling play a role (see [2], [6]). A frame for a vector space equipped with an inner product also allows each vector in the space to be written as a linear combination of the elements in the frame, but linear independence between the frame elements is not required. Intuitively, one can think about a frame as a basis to which one has added more elements (see [5]). One of the main purposes of this paper is to give a necessary and sufficient condition for a finite family of functions to constitute a Riesz family via linear independence of the

Copyright 2016 by the Tusi Mathematical Research Group.

Received Apr. 16, 2015; Accepted Aug. 15, 2015.

${ }^{*}$ Corresponding author.

2010 Mathematics Subject Classification. Primary 43A15; Secondary 43A25.

Keywords. Riesz family, locally compact Abelian group, uniform lattice, shifts of functions. 
Ann. Funct. Anal. 7 (2016), no. 2, 314-325

http://dx.doi.org/10.1215/20088752-3506016

ISSN: 2008-8752 (electronic)

ANNALS of

EUNCTIONAL

http://projecteuclid.org/afa

ANALYSIS

\title{
A CHARACTERIZATION OF THE RIESZ FAMILY OF SHIFTS OF FUNCTIONS ON LOCALLY COMPACT ABELIAN GROUPS
}

\author{
N. MOHAMMADIAN,${ }^{1 *}$ R. A. KAMYABI GOL,${ }^{2}$ and R. RAISI TOUSI ${ }^{2}$ \\ Communicated by V. Runde
}

\begin{abstract}
For a locally compact Abelian group $G$, we give a necessary and sufficient condition for shifts of a function $\phi \in L_{2}(G)$ to be a Riesz family. Also, for a finite family $\Phi$ of compactly supported functions in $L_{2}(G)$, we show that the shifts of $\Phi$ constitute a Riesz family if and only if the nets $(\phi(\xi \eta))_{\eta \in L^{\perp}}$, $\phi \in \Phi$, are linearly independent for all $\xi \in \hat{G}$.
\end{abstract}

\section{INTRODUCTION AND PRELIMINARIES}

Shift-invariant spaces and Riesz families play an increasingly important role in various areas of mathematical analysis and their applications. They appear in the study of spline wavelets, approximation, regular sampling, Gabor systems, and several others (see [1], [4], [8], [14]). A sequence $\left(x_{n}\right)_{n \in \mathbb{N}}$ for a Hilbert space $H$ is called a Riesz basis if it is a frame and is also a basis for $H$. Frames provide a useful tool to obtain signal decomposition in cases where redundancy, oversampling, and irregular sampling play a role (see [2], [6]). A frame for a vector space equipped with an inner product also allows each vector in the space to be written as a linear combination of the elements in the frame, but linear independence between the frame elements is not required. Intuitively, one can think about a frame as a basis to which one has added more elements (see [5]). One of the main purposes of this paper is to give a necessary and sufficient condition for a finite family of functions to constitute a Riesz family via linear independence of the

Copyright 2016 by the Tusi Mathematical Research Group.

Received Apr. 16, 2015; Accepted Aug. 15, 2015.

${ }^{*}$ Corresponding author.

2010 Mathematics Subject Classification. Primary 43A15; Secondary 43A25.

Keywords. Riesz family, locally compact Abelian group, uniform lattice, shifts of functions. 
Ann. Funct. Anal. 7 (2016), no. 2, 314-325

http://dx.doi.org/10.1215/20088752-3506016

ISSN: 2008-8752 (electronic)

ANNALS of

EUNCTIONAL

http://projecteuclid.org/afa

ANALYSIS

\title{
A CHARACTERIZATION OF THE RIESZ FAMILY OF SHIFTS OF FUNCTIONS ON LOCALLY COMPACT ABELIAN GROUPS
}

\author{
N. MOHAMMADIAN,${ }^{1 *}$ R. A. KAMYABI GOL,${ }^{2}$ and R. RAISI TOUSI ${ }^{2}$ \\ Communicated by V. Runde
}

\begin{abstract}
For a locally compact Abelian group $G$, we give a necessary and sufficient condition for shifts of a function $\phi \in L_{2}(G)$ to be a Riesz family. Also, for a finite family $\Phi$ of compactly supported functions in $L_{2}(G)$, we show that the shifts of $\Phi$ constitute a Riesz family if and only if the nets $(\phi(\xi \eta))_{\eta \in L^{\perp}}$, $\phi \in \Phi$, are linearly independent for all $\xi \in \hat{G}$.
\end{abstract}

\section{INTRODUCTION AND PRELIMINARIES}

Shift-invariant spaces and Riesz families play an increasingly important role in various areas of mathematical analysis and their applications. They appear in the study of spline wavelets, approximation, regular sampling, Gabor systems, and several others (see [1], [4], [8], [14]). A sequence $\left(x_{n}\right)_{n \in \mathbb{N}}$ for a Hilbert space $H$ is called a Riesz basis if it is a frame and is also a basis for $H$. Frames provide a useful tool to obtain signal decomposition in cases where redundancy, oversampling, and irregular sampling play a role (see [2], [6]). A frame for a vector space equipped with an inner product also allows each vector in the space to be written as a linear combination of the elements in the frame, but linear independence between the frame elements is not required. Intuitively, one can think about a frame as a basis to which one has added more elements (see [5]). One of the main purposes of this paper is to give a necessary and sufficient condition for a finite family of functions to constitute a Riesz family via linear independence of the

Copyright 2016 by the Tusi Mathematical Research Group.

Received Apr. 16, 2015; Accepted Aug. 15, 2015.

${ }^{*}$ Corresponding author.

2010 Mathematics Subject Classification. Primary 43A15; Secondary 43A25.

Keywords. Riesz family, locally compact Abelian group, uniform lattice, shifts of functions. 
Ann. Funct. Anal. 7 (2016), no. 2, 314-325

http://dx.doi.org/10.1215/20088752-3506016

ISSN: 2008-8752 (electronic)

ANNALS of

EUNCTIONAL

http://projecteuclid.org/afa

ANALYSIS

\title{
A CHARACTERIZATION OF THE RIESZ FAMILY OF SHIFTS OF FUNCTIONS ON LOCALLY COMPACT ABELIAN GROUPS
}

\author{
N. MOHAMMADIAN,${ }^{1 *}$ R. A. KAMYABI GOL,${ }^{2}$ and R. RAISI TOUSI ${ }^{2}$ \\ Communicated by V. Runde
}

\begin{abstract}
For a locally compact Abelian group $G$, we give a necessary and sufficient condition for shifts of a function $\phi \in L_{2}(G)$ to be a Riesz family. Also, for a finite family $\Phi$ of compactly supported functions in $L_{2}(G)$, we show that the shifts of $\Phi$ constitute a Riesz family if and only if the nets $(\phi(\xi \eta))_{\eta \in L^{\perp}}$, $\phi \in \Phi$, are linearly independent for all $\xi \in \hat{G}$.
\end{abstract}

\section{INTRODUCTION AND PRELIMINARIES}

Shift-invariant spaces and Riesz families play an increasingly important role in various areas of mathematical analysis and their applications. They appear in the study of spline wavelets, approximation, regular sampling, Gabor systems, and several others (see [1], [4], [8], [14]). A sequence $\left(x_{n}\right)_{n \in \mathbb{N}}$ for a Hilbert space $H$ is called a Riesz basis if it is a frame and is also a basis for $H$. Frames provide a useful tool to obtain signal decomposition in cases where redundancy, oversampling, and irregular sampling play a role (see [2], [6]). A frame for a vector space equipped with an inner product also allows each vector in the space to be written as a linear combination of the elements in the frame, but linear independence between the frame elements is not required. Intuitively, one can think about a frame as a basis to which one has added more elements (see [5]). One of the main purposes of this paper is to give a necessary and sufficient condition for a finite family of functions to constitute a Riesz family via linear independence of the

Copyright 2016 by the Tusi Mathematical Research Group.

Received Apr. 16, 2015; Accepted Aug. 15, 2015.

${ }^{*}$ Corresponding author.

2010 Mathematics Subject Classification. Primary 43A15; Secondary 43A25.

Keywords. Riesz family, locally compact Abelian group, uniform lattice, shifts of functions. 
Ann. Funct. Anal. 7 (2016), no. 2, 314-325

http://dx.doi.org/10.1215/20088752-3506016

ISSN: 2008-8752 (electronic)

ANNALS of

EUNCTIONAL

http://projecteuclid.org/afa

ANALYSIS

\title{
A CHARACTERIZATION OF THE RIESZ FAMILY OF SHIFTS OF FUNCTIONS ON LOCALLY COMPACT ABELIAN GROUPS
}

\author{
N. MOHAMMADIAN,${ }^{1 *}$ R. A. KAMYABI GOL,${ }^{2}$ and R. RAISI TOUSI ${ }^{2}$ \\ Communicated by V. Runde
}

\begin{abstract}
For a locally compact Abelian group $G$, we give a necessary and sufficient condition for shifts of a function $\phi \in L_{2}(G)$ to be a Riesz family. Also, for a finite family $\Phi$ of compactly supported functions in $L_{2}(G)$, we show that the shifts of $\Phi$ constitute a Riesz family if and only if the nets $(\phi(\xi \eta))_{\eta \in L^{\perp}}$, $\phi \in \Phi$, are linearly independent for all $\xi \in \hat{G}$.
\end{abstract}

\section{INTRODUCTION AND PRELIMINARIES}

Shift-invariant spaces and Riesz families play an increasingly important role in various areas of mathematical analysis and their applications. They appear in the study of spline wavelets, approximation, regular sampling, Gabor systems, and several others (see [1], [4], [8], [14]). A sequence $\left(x_{n}\right)_{n \in \mathbb{N}}$ for a Hilbert space $H$ is called a Riesz basis if it is a frame and is also a basis for $H$. Frames provide a useful tool to obtain signal decomposition in cases where redundancy, oversampling, and irregular sampling play a role (see [2], [6]). A frame for a vector space equipped with an inner product also allows each vector in the space to be written as a linear combination of the elements in the frame, but linear independence between the frame elements is not required. Intuitively, one can think about a frame as a basis to which one has added more elements (see [5]). One of the main purposes of this paper is to give a necessary and sufficient condition for a finite family of functions to constitute a Riesz family via linear independence of the

Copyright 2016 by the Tusi Mathematical Research Group.

Received Apr. 16, 2015; Accepted Aug. 15, 2015.

${ }^{*}$ Corresponding author.

2010 Mathematics Subject Classification. Primary 43A15; Secondary 43A25.

Keywords. Riesz family, locally compact Abelian group, uniform lattice, shifts of functions. 
Ann. Funct. Anal. 7 (2016), no. 2, 314-325

http://dx.doi.org/10.1215/20088752-3506016

ISSN: 2008-8752 (electronic)

ANNALS of

EUNCTIONAL

http://projecteuclid.org/afa

ANALYSIS

\title{
A CHARACTERIZATION OF THE RIESZ FAMILY OF SHIFTS OF FUNCTIONS ON LOCALLY COMPACT ABELIAN GROUPS
}

\author{
N. MOHAMMADIAN,${ }^{1 *}$ R. A. KAMYABI GOL,${ }^{2}$ and R. RAISI TOUSI ${ }^{2}$ \\ Communicated by V. Runde
}

\begin{abstract}
For a locally compact Abelian group $G$, we give a necessary and sufficient condition for shifts of a function $\phi \in L_{2}(G)$ to be a Riesz family. Also, for a finite family $\Phi$ of compactly supported functions in $L_{2}(G)$, we show that the shifts of $\Phi$ constitute a Riesz family if and only if the nets $(\phi(\xi \eta))_{\eta \in L^{\perp}}$, $\phi \in \Phi$, are linearly independent for all $\xi \in \hat{G}$.
\end{abstract}

\section{INTRODUCTION AND PRELIMINARIES}

Shift-invariant spaces and Riesz families play an increasingly important role in various areas of mathematical analysis and their applications. They appear in the study of spline wavelets, approximation, regular sampling, Gabor systems, and several others (see [1], [4], [8], [14]). A sequence $\left(x_{n}\right)_{n \in \mathbb{N}}$ for a Hilbert space $H$ is called a Riesz basis if it is a frame and is also a basis for $H$. Frames provide a useful tool to obtain signal decomposition in cases where redundancy, oversampling, and irregular sampling play a role (see [2], [6]). A frame for a vector space equipped with an inner product also allows each vector in the space to be written as a linear combination of the elements in the frame, but linear independence between the frame elements is not required. Intuitively, one can think about a frame as a basis to which one has added more elements (see [5]). One of the main purposes of this paper is to give a necessary and sufficient condition for a finite family of functions to constitute a Riesz family via linear independence of the

Copyright 2016 by the Tusi Mathematical Research Group.

Received Apr. 16, 2015; Accepted Aug. 15, 2015.

${ }^{*}$ Corresponding author.

2010 Mathematics Subject Classification. Primary 43A15; Secondary 43A25.

Keywords. Riesz family, locally compact Abelian group, uniform lattice, shifts of functions. 
Ann. Funct. Anal. 7 (2016), no. 2, 314-325

http://dx.doi.org/10.1215/20088752-3506016

ISSN: 2008-8752 (electronic)

ANNALS of

EUNCTIONAL

http://projecteuclid.org/afa

ANALYSIS

\title{
A CHARACTERIZATION OF THE RIESZ FAMILY OF SHIFTS OF FUNCTIONS ON LOCALLY COMPACT ABELIAN GROUPS
}

\author{
N. MOHAMMADIAN,${ }^{1 *}$ R. A. KAMYABI GOL,${ }^{2}$ and R. RAISI TOUSI ${ }^{2}$ \\ Communicated by V. Runde
}

\begin{abstract}
For a locally compact Abelian group $G$, we give a necessary and sufficient condition for shifts of a function $\phi \in L_{2}(G)$ to be a Riesz family. Also, for a finite family $\Phi$ of compactly supported functions in $L_{2}(G)$, we show that the shifts of $\Phi$ constitute a Riesz family if and only if the nets $(\phi(\xi \eta))_{\eta \in L^{\perp}}$, $\phi \in \Phi$, are linearly independent for all $\xi \in \hat{G}$.
\end{abstract}

\section{INTRODUCTION AND PRELIMINARIES}

Shift-invariant spaces and Riesz families play an increasingly important role in various areas of mathematical analysis and their applications. They appear in the study of spline wavelets, approximation, regular sampling, Gabor systems, and several others (see [1], [4], [8], [14]). A sequence $\left(x_{n}\right)_{n \in \mathbb{N}}$ for a Hilbert space $H$ is called a Riesz basis if it is a frame and is also a basis for $H$. Frames provide a useful tool to obtain signal decomposition in cases where redundancy, oversampling, and irregular sampling play a role (see [2], [6]). A frame for a vector space equipped with an inner product also allows each vector in the space to be written as a linear combination of the elements in the frame, but linear independence between the frame elements is not required. Intuitively, one can think about a frame as a basis to which one has added more elements (see [5]). One of the main purposes of this paper is to give a necessary and sufficient condition for a finite family of functions to constitute a Riesz family via linear independence of the

Copyright 2016 by the Tusi Mathematical Research Group.

Received Apr. 16, 2015; Accepted Aug. 15, 2015.

${ }^{*}$ Corresponding author.

2010 Mathematics Subject Classification. Primary 43A15; Secondary 43A25.

Keywords. Riesz family, locally compact Abelian group, uniform lattice, shifts of functions. 
Ann. Funct. Anal. 7 (2016), no. 2, 314-325

http://dx.doi.org/10.1215/20088752-3506016

ISSN: 2008-8752 (electronic)

ANNALS of

EUNCTIONAL

http://projecteuclid.org/afa

ANALYSIS

\title{
A CHARACTERIZATION OF THE RIESZ FAMILY OF SHIFTS OF FUNCTIONS ON LOCALLY COMPACT ABELIAN GROUPS
}

\author{
N. MOHAMMADIAN,${ }^{1 *}$ R. A. KAMYABI GOL,${ }^{2}$ and R. RAISI TOUSI ${ }^{2}$ \\ Communicated by V. Runde
}

\begin{abstract}
For a locally compact Abelian group $G$, we give a necessary and sufficient condition for shifts of a function $\phi \in L_{2}(G)$ to be a Riesz family. Also, for a finite family $\Phi$ of compactly supported functions in $L_{2}(G)$, we show that the shifts of $\Phi$ constitute a Riesz family if and only if the nets $(\phi(\xi \eta))_{\eta \in L^{\perp}}$, $\phi \in \Phi$, are linearly independent for all $\xi \in \hat{G}$.
\end{abstract}

\section{INTRODUCTION AND PRELIMINARIES}

Shift-invariant spaces and Riesz families play an increasingly important role in various areas of mathematical analysis and their applications. They appear in the study of spline wavelets, approximation, regular sampling, Gabor systems, and several others (see [1], [4], [8], [14]). A sequence $\left(x_{n}\right)_{n \in \mathbb{N}}$ for a Hilbert space $H$ is called a Riesz basis if it is a frame and is also a basis for $H$. Frames provide a useful tool to obtain signal decomposition in cases where redundancy, oversampling, and irregular sampling play a role (see [2], [6]). A frame for a vector space equipped with an inner product also allows each vector in the space to be written as a linear combination of the elements in the frame, but linear independence between the frame elements is not required. Intuitively, one can think about a frame as a basis to which one has added more elements (see [5]). One of the main purposes of this paper is to give a necessary and sufficient condition for a finite family of functions to constitute a Riesz family via linear independence of the

Copyright 2016 by the Tusi Mathematical Research Group.

Received Apr. 16, 2015; Accepted Aug. 15, 2015.

${ }^{*}$ Corresponding author.

2010 Mathematics Subject Classification. Primary 43A15; Secondary 43A25.

Keywords. Riesz family, locally compact Abelian group, uniform lattice, shifts of functions. 
Ann. Funct. Anal. 7 (2016), no. 2, 314-325

http://dx.doi.org/10.1215/20088752-3506016

ISSN: 2008-8752 (electronic)

ANNALS of

EUNCTIONAL

http://projecteuclid.org/afa

ANALYSIS

\title{
A CHARACTERIZATION OF THE RIESZ FAMILY OF SHIFTS OF FUNCTIONS ON LOCALLY COMPACT ABELIAN GROUPS
}

\author{
N. MOHAMMADIAN,${ }^{1 *}$ R. A. KAMYABI GOL,${ }^{2}$ and R. RAISI TOUSI ${ }^{2}$ \\ Communicated by V. Runde
}

\begin{abstract}
For a locally compact Abelian group $G$, we give a necessary and sufficient condition for shifts of a function $\phi \in L_{2}(G)$ to be a Riesz family. Also, for a finite family $\Phi$ of compactly supported functions in $L_{2}(G)$, we show that the shifts of $\Phi$ constitute a Riesz family if and only if the nets $(\phi(\xi \eta))_{\eta \in L^{\perp}}$, $\phi \in \Phi$, are linearly independent for all $\xi \in \hat{G}$.
\end{abstract}

\section{INTRODUCTION AND PRELIMINARIES}

Shift-invariant spaces and Riesz families play an increasingly important role in various areas of mathematical analysis and their applications. They appear in the study of spline wavelets, approximation, regular sampling, Gabor systems, and several others (see [1], [4], [8], [14]). A sequence $\left(x_{n}\right)_{n \in \mathbb{N}}$ for a Hilbert space $H$ is called a Riesz basis if it is a frame and is also a basis for $H$. Frames provide a useful tool to obtain signal decomposition in cases where redundancy, oversampling, and irregular sampling play a role (see [2], [6]). A frame for a vector space equipped with an inner product also allows each vector in the space to be written as a linear combination of the elements in the frame, but linear independence between the frame elements is not required. Intuitively, one can think about a frame as a basis to which one has added more elements (see [5]). One of the main purposes of this paper is to give a necessary and sufficient condition for a finite family of functions to constitute a Riesz family via linear independence of the

Copyright 2016 by the Tusi Mathematical Research Group.

Received Apr. 16, 2015; Accepted Aug. 15, 2015.

${ }^{*}$ Corresponding author.

2010 Mathematics Subject Classification. Primary 43A15; Secondary 43A25.

Keywords. Riesz family, locally compact Abelian group, uniform lattice, shifts of functions. 
Ann. Funct. Anal. 7 (2016), no. 2, 314-325

http://dx.doi.org/10.1215/20088752-3506016

ISSN: 2008-8752 (electronic)

ANNALS of

EUNCTIONAL

http://projecteuclid.org/afa

ANALYSIS

\title{
A CHARACTERIZATION OF THE RIESZ FAMILY OF SHIFTS OF FUNCTIONS ON LOCALLY COMPACT ABELIAN GROUPS
}

\author{
N. MOHAMMADIAN,${ }^{1 *}$ R. A. KAMYABI GOL,${ }^{2}$ and R. RAISI TOUSI ${ }^{2}$ \\ Communicated by V. Runde
}

\begin{abstract}
For a locally compact Abelian group $G$, we give a necessary and sufficient condition for shifts of a function $\phi \in L_{2}(G)$ to be a Riesz family. Also, for a finite family $\Phi$ of compactly supported functions in $L_{2}(G)$, we show that the shifts of $\Phi$ constitute a Riesz family if and only if the nets $(\phi(\xi \eta))_{\eta \in L^{\perp}}$, $\phi \in \Phi$, are linearly independent for all $\xi \in \hat{G}$.
\end{abstract}

\section{INTRODUCTION AND PRELIMINARIES}

Shift-invariant spaces and Riesz families play an increasingly important role in various areas of mathematical analysis and their applications. They appear in the study of spline wavelets, approximation, regular sampling, Gabor systems, and several others (see [1], [4], [8], [14]). A sequence $\left(x_{n}\right)_{n \in \mathbb{N}}$ for a Hilbert space $H$ is called a Riesz basis if it is a frame and is also a basis for $H$. Frames provide a useful tool to obtain signal decomposition in cases where redundancy, oversampling, and irregular sampling play a role (see [2], [6]). A frame for a vector space equipped with an inner product also allows each vector in the space to be written as a linear combination of the elements in the frame, but linear independence between the frame elements is not required. Intuitively, one can think about a frame as a basis to which one has added more elements (see [5]). One of the main purposes of this paper is to give a necessary and sufficient condition for a finite family of functions to constitute a Riesz family via linear independence of the

Copyright 2016 by the Tusi Mathematical Research Group.

Received Apr. 16, 2015; Accepted Aug. 15, 2015.

${ }^{*}$ Corresponding author.

2010 Mathematics Subject Classification. Primary 43A15; Secondary 43A25.

Keywords. Riesz family, locally compact Abelian group, uniform lattice, shifts of functions. 